\title{
BIEs for modelling of discontinuities in linear multi-field half-space
}

\author{
T. N. Pham ${ }^{1}$, J. Rungamornrat ${ }^{1}$, W. Pansuk ${ }^{1} \&$ Y. Sato ${ }^{2}$ \\ ${ }^{1}$ Department of Civil Engineering, Faculty of Engineering, \\ Chulalongkorn University, Thailand \\ ${ }^{2}$ Department of Civil Engineering, Faculty of Engineering, \\ Hokkaido University, Japan
}

\begin{abstract}
This paper presents an efficient and accurate boundary integral equation method for solving a linear, multi-field, half-space containing a surface of discontinuity and subjected to symmetrical and anti-symmetrical conditions on the boundary. Responses of the half-space are governed by a set of linear partial differential equations which are formulated in a general framework allowing the treatment of Laplace equation, linear elasticity problems, and problems involving multi-field materials such as piezoelectric, piezomagnetic, and piezoelectromagnetic solids. In the formulation, a systematic regularization procedure via the integration by parts, symmetrical and anti-symmetrical properties, and special representations of strongly singular and hyper singular kernels is employed to derive a set of singularity-reduced boundary integral relations. A pair of weak-form boundary integral equations involving both the sum and relative crack-face state variable and surface flux across the discontinuity surface is finally established and they contain only weakly singular kernels. A standard symmetric Galerkin boundary element method (SGBEM) is then implemented to solve those weakly singular integral equations for unknown data on the discontinuity surface. In numerical implementations, continuous local interpolation functions are employed in the approximation of solutions and an efficient means for both the kernel evaluation and the numerical integration is adopted to enhance the accuracy and computational efficiency of the developed scheme. The proposed numerical technique is then verified with various, reliable benchmark cases and a selected set of results is presented to demonstrate its capability and robustness.
\end{abstract}

Keywords: half-space, discontinuity, SGBEM, SIFs, T-stress, weakly singular. 


\section{Introduction}

A half-space containing a surface of discontinuities is one of mathematical domains commonly used to represent a physical body in the simulation of localized, near-surface flaws, defects, and impurities if their sizes are sufficiently small in comparison with the characteristic dimensions of the body. Such idealization significantly simplifies the real geometry, reduces the computational effort, and also yields the reasonably accurate prediction relative to the full analysis of the whole body. Various investigators employed the classical theory of linear elasticity and the concept of linear fracture mechanics along with either analytical or numerical solution procedures to model, simulate, and solve cracked half-spaces under various scenarios (e.g. [1-6, 9-12, 17]). Among various existing techniques, ones based on boundary integral equations have been found attractive and extensively employed in the analysis procedure due to their simplicity to treat the unbounded geometry (e.g. $[3,5,6,9,17])$. With the proper incorporation of fundamental solutions to satisfy the conditions on the free surface, the key governing integral equations simply involve unknowns on the discontinuity surface and this, as a result, significantly reduces the number of degrees of freedom resulting from the discretization (e.g. [6]). However, the strong smoothness requirement of the crack-face data due to the presence of strongly singular and hyper singular kernels still poses the difficulty on most of conventional boundary element methods. An attractive alternative to circumvent such difficulty is to employ singularity-reduced boundary integral equations that contain kernels of weaker singularity and require less smooth crack-face data. Li [6] successfully derived weakly singular integral equations for cracks in an elastic half space under various boundary conditions. However, his formulation was limited to an isotropic case and the implementation of those equations to solve half-space problems has not been recognized. The regularization procedure analogous to that used by Li [6] was extended to model cracks in a whole space and finite bodies [7, $8]$ and to take into account the material anisotropy [13, 14], but the extension for the case of a half-space has not been found. In the present study, the procedure used by $[6,7,13-15]$ is further generalized to derive a set of regularized integral equations for a half-space containing discontinuities and made of multi-field materials. In addition, a solution scheme based on a well known, weakly singular boundary integral equation method is also established.

\section{Problem description}

Consider a half-space $\Omega \subset \mathbb{R}^{3}$ containing embedded and surface breaking discontinuities as shown schematically in Figure 1. The surface of discontinuities in the reference state can be represented by two geometrically identical surfaces $S^{+}$and $S^{-}$with the corresponding outward unit normal vectors $\boldsymbol{n}^{+}$and $\boldsymbol{n}^{-}$, respectively. For convenience in the later development, a reference Cartesian coordinate system $\left(\boldsymbol{O} ; x_{1}, x_{2}, x_{3}\right)$ with the orthonormal base vectors $\left\{\boldsymbol{e}_{1}, \boldsymbol{e}_{2}, \boldsymbol{e}_{3}\right\}$ is chosen such that the origin $\boldsymbol{O}$ is located on the free surface; the $x_{3}$-axis directs downward; and the $x_{1}$ - and $x_{2}$-axes follow the right hand rule. 


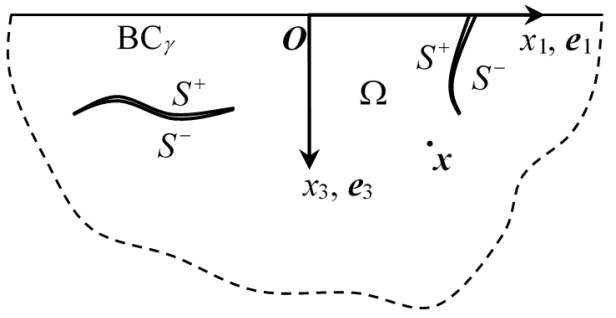

Figure 1: Schematic of a half-space containing isolated discontinuities and subjected to boundary condition of the type $\mathrm{BC}_{\gamma}$ on the free surface.

A state of the half-space under the action of external excitations is assumed completely described by $\alpha$ vector fields $\boldsymbol{u}^{(1)}, \boldsymbol{u}^{(2)}, \ldots, \boldsymbol{u}^{(\alpha)}$ and $\beta$ scalar fields $\phi^{[1]}, \phi^{[2]}, \ldots, \phi^{[\beta]}$, which are termed the "state variable". The spatial variation of the state variable is measured by $\alpha$ symmetric, second-order, tensor fields $\boldsymbol{\varepsilon}^{(1)}, \boldsymbol{\varepsilon}^{(2)}, \ldots, \boldsymbol{\varepsilon}^{(\alpha)}$ and $\beta$ vector fields $\boldsymbol{g}^{[1]}, \boldsymbol{g}^{[2]}, \ldots, \boldsymbol{g}^{[\beta]}$, and they are directly related to the state variable by

$$
\varepsilon_{i j}^{(k)}=\frac{1}{2}\left(u_{i, j}^{(k)}+u_{j, i}^{(k)}\right), \quad g_{i}^{[k]}=\phi_{, i}^{[k]}
$$

where a symbol $f_{, i}$ is used to represent a partial derivative of a function $f$ with respect to the coordinate $x_{i}$. Here and in what follows, lower-case indices, lowercases indices with the parenthesis, and lower-case indices with the bracket range from 1 to 3,1 to $\alpha$, and 1 to $\beta$, respectively, and the repeated indices imply the summation over their range. Now, the flux within the body used to measure the internal responses induced by the external disturbance and commonly termed the "body flux" can be described by $\alpha$ symmetric, second-order, tensor fields $\boldsymbol{\sigma}^{(1)}, \boldsymbol{\sigma}^{(2)}, \ldots, \boldsymbol{\sigma}^{(\alpha)}$ and $\beta$ vector fields $\boldsymbol{s}^{[1]}, \boldsymbol{s}^{[2]}, \ldots, \boldsymbol{s}^{[\beta]}$. The body flux is related to the gradient of the state variable via the following linear constitutive relations:

$$
\sigma_{i j}^{(k)}=E_{i j p q}^{(k)(r)} \varepsilon_{p q}^{(r)}+D_{i j p}^{(k)[r]} g_{p}^{[r]}, \quad s_{i}^{[k]}=D_{p q i}^{[k](r)} \varepsilon_{p q}^{(r)}+C_{i p}^{[k][r]} g_{p}^{[r]}
$$

where $E_{i j p q}^{(k)(r)}, D_{i j p}^{(k)[r]}$, and $C_{i p}^{[k][r]}$ are fourth-, third-, and second-order tensors containing material constants. In the present study, materials are limited to those satisfying following symmetries: $E_{i j p q}^{(k)(r)}=E_{j i p q}^{(k)(r)}=E_{i j q p}^{(k)(r)}=E_{p q i j}^{(k)(r)}, E_{i j p q}^{(k)(r)}=E_{i j p q}^{(r)(k)}$, $D_{i j p}^{(k)[r]}=D_{j i p}^{(k)[r]}, D_{i j p}^{[k](r)}=D_{j i p}^{[k](r)}, D_{i j p}^{(k)[r]}=D_{i j p}^{[r](k)}, C_{i p}^{[k][r]}=C_{p i}^{[k][r]}, C_{i p}^{[k][r]}=C_{i p}^{[r][k]}$ and, in addition, the $x_{1}-x_{2}$ plane is the plane of material symmetry. Besides (2), the body flux is also related to the applied distributed body source, denoted by $\alpha$ vector fields $\boldsymbol{b}^{(1)}, \boldsymbol{b}^{(2)}, \ldots, \boldsymbol{b}^{(\alpha)}$ and $\beta$ scalar fields $a^{[1]}, a^{[2]}, \ldots, a^{[\beta]}$, by

$$
\sigma_{j i, j}^{(k)}+b_{i}^{(k)}=0, \quad s_{i, i}^{[k]}+a^{[k]}=0
$$


The surface flux at any point on an oriented, smooth surface with the corresponding unit normal vector $\boldsymbol{n}$, denoted by $\alpha$ vectors $\boldsymbol{t}^{(1)}, \boldsymbol{t}^{(2)}, \ldots, \boldsymbol{t}^{(\alpha)}$ and $\beta$ scalars $d^{[1]}, d^{[2]}, \ldots, d^{[\beta]}$, is related to the body flux by

$$
t_{i}^{(k)}=\sigma_{j i}^{(k)} n_{j}, \quad d^{[k]}=s_{j}^{[k]} n_{j}
$$

A set of basic field equations shown above for multi-field problems can be applied to various cases by first setting the value of the integers $\alpha$ and $\beta$ and then properly defining the field quantities and material constants to match those of each particular problem. For instance, it is applicable to steady-state heat conduction problems, steady-state flows in porous media, and Laplace equation for $\alpha=0$ and $\beta=1$, to linear elasticity problems for $\alpha=1$ and $\beta=0$, to linear piezoelectric and linear piezomagnetic problems for $\alpha=1$ and $\beta=1$, and to linear piezoelectromagnetic problems for $\alpha=1$ and $\beta=2$. For conciseness of representations, we define a single $(3 \alpha+\beta)$-component vector $\hat{\boldsymbol{u}}$ containing all components of the state variable $\boldsymbol{u}^{(1)}, \boldsymbol{u}^{(2)}, \ldots, \boldsymbol{u}^{(\alpha)}$ and $\phi^{[1]}, \phi^{[2]}, \ldots, \phi^{[\beta]}$ such that $u_{i}^{(k)}$ is stored at $\hat{u}_{3(k)+i-3}$ and $\phi^{[k]}$ is stored at $\hat{u}_{3 \alpha+[k]}$ and a single $3 \mathrm{x}(3 \alpha+\beta)$ matrix $\hat{\sigma}$ containing all components of the body flux $\sigma^{(1)}, \sigma^{(2)}, \ldots, \sigma^{(\alpha)}$ and $s^{[1]}, s^{[2]}, \ldots, s^{[\beta]}$ such that $\sigma_{i j}^{(k)}$ is stored at $\hat{\sigma}_{i, 3(k)+j-3}$ and $\sigma_{i}^{[k]}$ is stored at $\hat{\sigma}_{i, 3 \alpha+[k]}$. With this definition, (3) and the combination of (1) and (2) take a form:

$$
\hat{\sigma}_{i J, i}+\hat{b}_{J}=0 ; \quad \hat{\sigma}_{i J}=\hat{E}_{i J K m} \hat{u}_{K, m}
$$

where $\hat{b}_{J}$ denotes the "distributed body source" obtained from $\boldsymbol{b}^{(1)}, \boldsymbol{b}^{(2)}, \ldots, \boldsymbol{b}^{(\alpha)}$ and $a^{[1]}, a^{[2]}, \ldots, a^{[\beta]}$ in the manner consistent with $\hat{\sigma}_{i J} ; \hat{E}_{i J K m}$ is termed the "generalized moduli" obtained from $E_{i j p q}^{(k)(r)}, D_{i j p}^{(k)[r]}$, and $C_{i p}^{[k][r]}$ in the manner consistent with $\hat{\sigma}_{i J}$; and, here and in what follows, upper case indices range from 1 to $(3 \alpha+\beta)$ and repeated indices imply the summation over their range. It is worth noting that due to the symmetry of $E_{i j p q}^{(k)(r)}, D_{i j p}^{(k)[r]}$ and $C_{i p}^{[k][r]}, \hat{E}_{i J K m}$ obviously satisfies $\hat{E}_{i J K m}=\hat{E}_{m K J i}$. Similarly, the surface flux $\boldsymbol{t}^{(1)}, \boldsymbol{t}^{(2)}, \ldots, \boldsymbol{t}^{(\alpha)}$ and $d^{[1]}, d^{[2]}, \ldots, d^{[\beta]}$ can be stored in a single $(3 \alpha+\beta)$-component vector $\hat{\boldsymbol{t}}$ such that $t_{i}^{(k)}$ and $d^{[k]}$ are stored at $\hat{t}_{3(k)+i-3}$ and $\hat{t}_{3 \alpha+[k]}$, respectively. As a result, components of $\hat{\boldsymbol{t}}$ are given by $\hat{t}_{J}=\hat{\sigma}_{i J} n_{i}$. In the present study, material properties are assumed homogeneous; the distributed body source and the remote excitation identically vanish; and the surface of discontinuities is sufficiently smooth. On $x_{3}=0$, the half-space is subjected to either a symmetrical boundary condition (termed $\mathrm{BC}_{1}$ ) or an anti-symmetrical boundary condition (termed $\mathrm{BC}_{2}$ ); in particular, the conditions $\hat{t}_{3(k)-2}=\hat{t}_{3(k)-1}=\hat{u}_{3(k)}=\hat{u}_{3 \alpha+[k]}=0$ and the conditions $\hat{u}_{3(k)-2}=\hat{u}_{3(k)-1}=\hat{t}_{3(k)}=\hat{t}_{3 \alpha+[k]}=0$ are satisfied on $x_{3}=0$ for $\mathrm{BC}_{1}$ and $\mathrm{BC}_{2}$, respectively. 


\section{Fundamental solutions of half-space}

Fundamental solutions of both the state variable and the body flux for a half-space under $\mathrm{BC}_{\gamma}$ with $\gamma=1,2$ can be constructed using the existing general fundamental solutions of the whole space (e.g. [15]) along with the symmetrical and antisymmetrical conditions. It should be emphasized that results presented here are limited only to the case that a material constituting the half-space is homogeneous and possesses the plane $x_{3}=0$ as a plane of material symmetry.

Now, consider a half-space subjected to boundary conditions of the type $\mathrm{BC}_{\gamma}$ (for $\gamma=1,2$ ) and a unit concentrated source $\hat{\boldsymbol{b}}^{M}$ (with their components defined by $\hat{b}_{J}^{M}(\boldsymbol{\xi})=\delta_{J M} \delta(\boldsymbol{\xi}-\boldsymbol{x})$ ) at a source point $\boldsymbol{x}=x_{k} \boldsymbol{e}_{k}$ as shown in Figure 2(a) where $\delta_{J M}$ denotes the Kronecker-delta symbol and $\delta(\boldsymbol{\xi}-\boldsymbol{x})$ is the Dirac-delta distribution centered at $\boldsymbol{x}$. This half-space is identical to the bottom half of the whole space subjected to a unit concentrated source $\hat{\boldsymbol{b}}^{M}$ at a point $\boldsymbol{x}$ and a unit concentrated source $\hat{\boldsymbol{b}}^{\gamma M}$ at a point $\overline{\boldsymbol{x}}=\bar{x}_{k} \boldsymbol{e}_{k}$ with its components defined by $\hat{b}_{J}^{\gamma M}(\boldsymbol{\xi})=\Lambda_{\gamma} \bar{\delta}_{J M} \delta(\boldsymbol{\xi}-\overline{\boldsymbol{x}})$ as illustrated schematically in Figure 2(b) where $\bar{x}_{k}=\bar{\delta}_{k p} x_{p}, \bar{\delta}_{11}=\bar{\delta}_{22}=-\bar{\delta}_{33}=1$ and $\bar{\delta}_{i j}=0, i \neq j ; \Lambda_{1}=1$ and $\Lambda_{2}=-1 ;$ the symbol $\bar{\delta}_{J M}$ is defined such that $\bar{\delta}_{J M}=0$ for $J \neq M, \bar{\delta}_{J M}=-1$ for $J=M=3(k), 3 \alpha+[k]$, otherwise $\bar{\delta}_{J M}=1$; and Greek indices range from 1 to 2 . Upon using both symmetry and anti-symmetry, fundamental solutions for state variable and body flux, denoted respectively by $U_{J}^{\gamma P}(\boldsymbol{\xi}, \boldsymbol{x})$ and $S_{i J}^{\gamma P}(\boldsymbol{\xi}, \boldsymbol{x})$, can be obtained as

$$
\begin{aligned}
& U_{J}^{\gamma P}(\boldsymbol{\xi}, \boldsymbol{x})=\Gamma_{\gamma} U_{J}^{P}(\boldsymbol{\xi}-\boldsymbol{x})+\Lambda_{\gamma} \bar{\delta}_{P K} U_{J}^{K}(\boldsymbol{\xi}-\overline{\boldsymbol{x}}) \\
& S_{i J}^{\gamma P}(\boldsymbol{\xi}, \boldsymbol{x})=\Gamma_{\gamma} S_{i J}^{P}(\boldsymbol{\xi}-\boldsymbol{x})+\Lambda_{\gamma} \bar{\delta}_{P K} S_{i J}^{K}(\boldsymbol{\xi}-\overline{\boldsymbol{x}})
\end{aligned}
$$

where $\Gamma_{1}=\Gamma_{2}=1$ and $U_{J}^{P}(\boldsymbol{\xi}-\boldsymbol{x})$ and $S_{i J}^{P}(\boldsymbol{\xi}-\boldsymbol{x})$ are fundamental solutions of the state variable and the body flux for a whole space under a unit concentrated source $\hat{b}_{J}^{P}(\boldsymbol{\xi})=\delta_{J P} \delta(\boldsymbol{\xi}-\boldsymbol{x})$. The explicit form of $U_{J}^{P}(\boldsymbol{\xi}-\boldsymbol{x})$ and $S_{i J}^{P}(\boldsymbol{\xi}-\boldsymbol{x})$ for certain classes of multi-field materials can be found in the work of [15]. Due to the singularity nature of $U_{J}^{P}(\boldsymbol{\xi}-\boldsymbol{x})$ and $S_{i J}^{P}(\boldsymbol{\xi}-\boldsymbol{x}), U_{J}^{\gamma P}(\boldsymbol{\xi}, \boldsymbol{x})$ and $S_{i J}^{\gamma P}(\boldsymbol{\xi}, \boldsymbol{x})$ are singular at $\boldsymbol{\xi}=\boldsymbol{x}$ of $\mathcal{O}(1 / r)$ and $\mathcal{O}\left(1 / r^{2}\right)$ where $r=\|\boldsymbol{\xi}-\boldsymbol{x}\|$, respectively.

\section{Formulation of weakly singular integral equations}

By generalizing Somigliana's identity to a half-space containing the surface of discontinuities and subjected to $\mathrm{BC}_{\gamma}$ along with utilizing the fundamental solutions established in the previous section, it leads to a boundary integral relation for the state variable

$$
\hat{u}_{P}^{\gamma}(\boldsymbol{x})=\int_{S^{+}} U_{J}^{\gamma P}(\boldsymbol{\xi}, \boldsymbol{x}) \sum \hat{t}_{J}(\boldsymbol{\xi}) d A(\boldsymbol{\xi})-\int_{S^{+}} S_{i J}^{\gamma P}(\boldsymbol{\xi}, \boldsymbol{x}) n_{i}^{+}(\boldsymbol{\xi}) \Delta \hat{u}_{J}(\boldsymbol{\xi}) d A(\boldsymbol{\xi})
$$




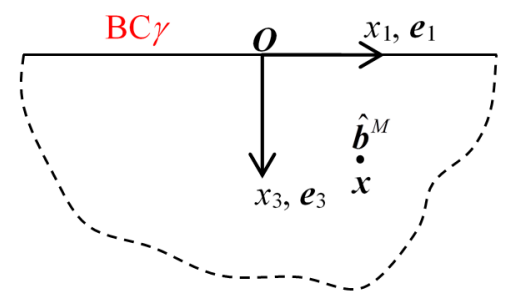

(a)

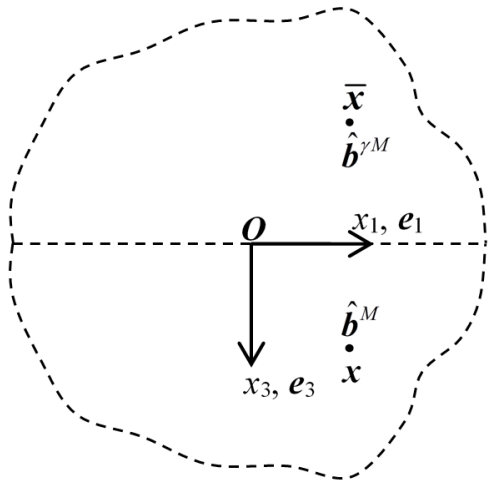

(b)

Figure 2: $\quad$ Schematics of (a) half-space subjected to $\mathrm{BC}_{\gamma}$ and unit concentrated source $\hat{\boldsymbol{b}}^{M}$ at $\boldsymbol{x}$ and (b) whole space subjected to unit concentrated source $\hat{\boldsymbol{b}}^{M}$ at $\boldsymbol{x}$ and unit concentrated source $\hat{\boldsymbol{b}}^{\gamma M}$ at its image point $\overline{\boldsymbol{x}}$.

where $\boldsymbol{x}$ is any interior point of the half-space; $\Sigma \hat{t}_{J}(\boldsymbol{\xi}) \equiv \hat{t}_{J}\left(\xi^{+}\right)+\hat{t}_{J}\left(\boldsymbol{\xi}^{-}\right)$and $\Delta \hat{u}_{J}(\xi) \equiv \hat{u}_{J}\left(\xi^{+}\right)-\hat{u}_{J}\left(\xi^{-}\right)$denotes the sum of the surface flux and the jump in the state variable across $S^{+}$and $S^{-}$, respectively; and $\xi^{+} \in S^{+}$and $\xi^{-} \in S^{-}$are two coincident points on the surface of discontinuities. It should be remarked that the reduction to integrals over a single surface $S^{+}$results directly from the continuity of the fundamental solutions $U_{J}^{\gamma P}(\boldsymbol{\xi}, \boldsymbol{x})$ and $S_{i J}^{\gamma P}(\boldsymbol{\xi}, \boldsymbol{x})$ at the field point $\boldsymbol{\xi}$. By first computing the derivative of (8) to obtain the spatial gradient of $\hat{u}_{P}^{\gamma}$ with respect to $x_{i}$ and then employing the constitutive relation (5), it yields a boundary integral relation for the body flux

$$
\sigma_{I K}^{\gamma}(\boldsymbol{x})=\int_{S^{+}} E_{l K P q} \frac{\partial U_{J}^{\gamma P}(\boldsymbol{\xi}, \boldsymbol{x})}{\partial x_{q}} \Sigma \hat{t}_{J}(\boldsymbol{\xi}) d A(\boldsymbol{\xi})-\int_{S^{+}} E_{l K P q} \frac{\partial S_{i J}^{\gamma P}(\boldsymbol{\xi}, \boldsymbol{x})}{\partial x_{q}} n_{i}^{+}(\boldsymbol{\xi}) \Delta \hat{u}_{J}(\boldsymbol{\xi}) d A(\boldsymbol{\xi})
$$

From the relation (7) along with the properties of the fundamental solutions $U_{J}^{P}(\boldsymbol{\xi}-\boldsymbol{x})$ and $S_{i J}^{P}(\boldsymbol{\xi}-\boldsymbol{x})$ for the whole space, the kernels $E_{l K P q} \partial U_{J}^{\gamma P}(\boldsymbol{\xi}, \boldsymbol{x}) / \partial x_{q}$ and $E_{l K P q} \partial S_{i J}^{\gamma P}(\boldsymbol{\xi}, \boldsymbol{x}) / \partial x_{q}$ appearing in the integral relation (9) can be obtained as

$$
\begin{gathered}
E_{l K P q} \frac{\partial U_{J}^{\gamma P}(\boldsymbol{\xi}, \boldsymbol{x})}{\partial x_{q}}=-\Gamma_{\gamma} S_{l K}^{J}(\boldsymbol{\xi}-\boldsymbol{x})+\Lambda_{\gamma} \bar{\delta}_{J P} S_{l K}^{P}(\boldsymbol{x}-\overline{\boldsymbol{\xi}}) \\
E_{l K P q} \frac{\partial S_{i J}^{\gamma P}(\boldsymbol{\xi}, \boldsymbol{x})}{\partial x_{q}}=-\Gamma_{\gamma} \Sigma_{i J}^{l K}(\boldsymbol{\xi}-\boldsymbol{x})-\Lambda_{\gamma} \bar{\delta}_{i a} \bar{\delta}_{J B} \Sigma_{a B}^{l K}(\boldsymbol{x}-\overline{\boldsymbol{\xi}})
\end{gathered}
$$


where $\Sigma_{i J}^{I K}(\boldsymbol{\xi}-\boldsymbol{x})=E_{I K P q} \partial S_{i J}^{P}(\boldsymbol{\xi}-\boldsymbol{x}) / \partial \xi_{q}$. From the singularity of the function $S_{i J}^{P}(\boldsymbol{\xi}-\boldsymbol{x})$, the kernels $E_{l K P q} \partial U_{J}^{\gamma P}(\boldsymbol{\xi}, \boldsymbol{x}) / \partial x_{q}$ and $E_{l K P q} \partial S_{i J}^{\gamma P}(\boldsymbol{\xi}, \boldsymbol{x}) / \partial x_{q}$ are clearly singular at $\boldsymbol{\xi}=\boldsymbol{x}$ of $\mathcal{O}\left(1 / r^{2}\right)$ and $\mathcal{O}\left(1 / r^{3}\right)$, respectively. By taking the limit of the boundary integral relation (8) to any point on the discontinuity surface, it yields a standard boundary integral equation for the sum of the state variable on the discontinuity surface. However, such integral equation is mathematically degenerate and not sufficient for solving all primary unknowns on the surface of discontinuities. To circumvent this difficulty, the boundary integral equation for the jump in the surface flux across the surface of discontinuities is employed. Such the integral equation can be derived from the boundary integral relation of the body flux (9) by taking the limit to any point on the surface $S^{+}$. However, such conventional surface-flux integral equation still contains strongly singular and hyper singular kernels and requires some special numerical treatments.

To aid the regularization of the boundary integral relations (8) and (9), three kernels $S_{i J}^{\gamma P}(\boldsymbol{\xi}, \boldsymbol{x}), E_{I K P q} \partial U_{J}^{\gamma P}(\boldsymbol{\xi}, \boldsymbol{x}) / \partial x_{q}$ and $E_{l K P q} \partial S_{i J}^{\gamma P}(\boldsymbol{\xi}, \boldsymbol{x}) / \partial x_{q}$ are decomposed into those suiting the integration by parts via Stokes' theorem. The key component to achieve this essential task is based on the special decompositions of the kernels $U_{J}^{P}(\boldsymbol{\xi}-\boldsymbol{x})$ and $S_{i J}^{P}(\boldsymbol{\xi}-\boldsymbol{x})$ for the whole space proposed by [15]:

$$
\begin{gathered}
S_{i J}^{P}(\boldsymbol{\xi}-\boldsymbol{x})=H_{i J}^{P}(\boldsymbol{\xi}-\boldsymbol{x})+\varepsilon_{i s m} \frac{\partial}{\partial \xi_{s}} G_{m J}^{P}(\boldsymbol{\xi}-\boldsymbol{x}) \\
\Sigma_{i J}^{I K}(\boldsymbol{\xi}-\boldsymbol{x})=-E_{i J K l} \delta(\boldsymbol{\xi}-\boldsymbol{x})+\varepsilon_{i s m} \frac{\partial}{\partial \xi_{s}} \varepsilon_{l r t} \frac{\partial}{\partial \xi_{r}} C_{m J}^{t K}(\boldsymbol{\xi}-\boldsymbol{x})
\end{gathered}
$$

where $\varepsilon_{i s m}$ is an alternating symbol; $H_{i J}^{P}(\boldsymbol{\xi}-\boldsymbol{x})=-\delta_{J P}\left(\xi_{i}-x_{i}\right) / 4 \pi r^{3}$; and $G_{m J}^{P}(\boldsymbol{\xi}-\boldsymbol{x})$ and $C_{m J}^{t K}(\boldsymbol{\xi}-\boldsymbol{x})$ are weakly singular at $\boldsymbol{\xi}=\boldsymbol{x}$ of $\mathcal{O}(1 / r)$. By using (7), (10) and (11), and the decompositions (12) and (13), the kernels $S_{i J}^{\gamma P}(\boldsymbol{\xi}, \boldsymbol{x})$, $E_{I K P q} \partial U_{J}^{\gamma P}(\boldsymbol{\xi}, \boldsymbol{x}) / \partial x_{q}$ and $E_{I K P q} \partial S_{i J}^{\gamma P}(\boldsymbol{\xi}, \boldsymbol{x}) / \partial x_{q}$ admit the representations

$$
\begin{gathered}
S_{i J}^{\gamma P}(\boldsymbol{\xi}-\boldsymbol{x})=H_{i J}^{\gamma P}(\boldsymbol{\xi}, \boldsymbol{x})+\varepsilon_{i s m} \frac{\partial}{\partial \xi_{s}} G_{m J}^{\gamma P}(\boldsymbol{\xi}, \boldsymbol{x}) \\
E_{l K P q} \frac{\partial U_{J}^{\gamma P}(\boldsymbol{\xi}, \boldsymbol{x})}{\partial x_{q}}=H_{l K}^{\gamma J}(\boldsymbol{x}, \boldsymbol{\xi})+\varepsilon_{l r t} \frac{\partial}{\partial x_{r}} G_{t K}^{\gamma J}(\boldsymbol{x}, \boldsymbol{\xi}) \\
E_{l K P q} \frac{\partial S_{i J}^{\gamma P}(\boldsymbol{\xi}, \boldsymbol{x})}{\partial x_{q}}=\Gamma_{\gamma} E_{i J K l} \delta(\boldsymbol{\xi}-\boldsymbol{x})-\Lambda_{\gamma} \bar{\delta}_{i a} \bar{\delta}_{J P} E_{a P K l} \delta(\boldsymbol{x}-\overline{\boldsymbol{\xi}})+\varepsilon_{i s m} \frac{\partial}{\partial \xi_{s}} \varepsilon_{l r t} \frac{\partial}{\partial x_{r}} C_{m J}^{\gamma t K}(\boldsymbol{\xi}, \boldsymbol{x})
\end{gathered}
$$

where the functions $H_{i J}^{\gamma P}(\boldsymbol{\xi}, \boldsymbol{x}), G_{m J}^{\gamma P}(\boldsymbol{\xi}, \boldsymbol{x})$ and $C_{m J}^{\gamma t K}(\boldsymbol{\xi}, \boldsymbol{x})$ are defined by

$$
\begin{aligned}
& H_{i J}^{\gamma P}(\boldsymbol{\xi}, \boldsymbol{x})=\Gamma_{\gamma} H_{i J}^{P}(\boldsymbol{\xi}-\boldsymbol{x})+\Lambda_{\gamma} \bar{\delta}_{P K} H_{i J}^{K}(\boldsymbol{\xi}-\overline{\boldsymbol{x}}) \\
& G_{m J}^{\gamma P}(\boldsymbol{\xi}, \boldsymbol{x})=\Gamma_{\gamma} G_{m J}^{P}(\boldsymbol{\xi}-\boldsymbol{x})+\Lambda_{\gamma} \bar{\delta}_{P K} G_{m J}^{K}(\boldsymbol{\xi}-\overline{\boldsymbol{x}})
\end{aligned}
$$




$$
C_{m J}^{\gamma t K}(\boldsymbol{\xi}, \boldsymbol{x})=\Gamma_{\gamma} C_{m J}^{t K}(\boldsymbol{\xi}-\boldsymbol{x})-\Lambda_{\gamma} \bar{\delta}_{m a} \bar{\delta}_{J P} C_{a P}^{t K}(\boldsymbol{x}-\overline{\boldsymbol{\xi}})
$$

It is remarked that the kernels $H_{i J}^{\gamma P}(\boldsymbol{\xi}, \boldsymbol{x}), G_{m J}^{\gamma P}(\boldsymbol{\xi}, \boldsymbol{x})$, and $C_{m J}^{\gamma t K}(\boldsymbol{\xi}, \boldsymbol{x})$ are singular at $\boldsymbol{\xi}=\boldsymbol{x}$ of $\mathcal{O}\left(1 / r^{2}\right), \mathcal{O}(1 / r)$ and $\mathcal{O}(1 / r)$, respectively.

To establish the singularity-reduced integral relations for the state variable and the body flux, the special decompositions (14)-(16) are first applied to the boundary integral relations (8) and (9). Then, certain integrals are integrated by parts via Stokes' theorem. The final integral relations are given by

$$
\begin{aligned}
\hat{u}_{P}^{\gamma}(\boldsymbol{x}) & =\int_{S^{+}} U_{J}^{\gamma P}(\boldsymbol{\xi}, \boldsymbol{x}) \sum \hat{t}_{J}(\boldsymbol{\xi}) d A(\boldsymbol{\xi})-\int_{S^{+}} H_{i J}^{\gamma P}(\boldsymbol{\xi}, \boldsymbol{x}) n_{i}^{+}(\boldsymbol{\xi}) \Delta \hat{u}_{J}(\boldsymbol{\xi}) d A(\boldsymbol{\xi}) \\
& +\int_{S^{+}} G_{m J}^{\gamma P}(\boldsymbol{\xi}, \boldsymbol{x}) D_{m} \Delta \hat{u}_{J}(\boldsymbol{\xi}) d A(\boldsymbol{\xi})-\int_{\partial S^{+}} G_{m J}^{\gamma P}(\boldsymbol{\xi}, \boldsymbol{x}) \Delta \hat{u}_{J}(\boldsymbol{\xi}) d \xi_{m} \\
\sigma_{l K}^{\gamma}(\boldsymbol{x})= & \varepsilon_{l r t} \frac{\partial}{\partial x_{r}}\left\{\int_{S^{+}} C_{m J}^{\gamma t K}(\boldsymbol{\xi}, \boldsymbol{x}) D_{m} \Delta \hat{u}_{J}(\boldsymbol{\xi}) d A(\boldsymbol{\xi})+\int_{S^{+}} G_{t K}^{\gamma J}(\boldsymbol{x}, \boldsymbol{\xi}) \sum \hat{t}_{J}(\boldsymbol{\xi}) d A(\boldsymbol{\xi})\right\} \\
& -\varepsilon_{l r t} \frac{\partial}{\partial x_{r}}\left\{\int_{\partial S^{+}} C_{m J}^{\gamma t K}(\boldsymbol{\xi}, \boldsymbol{x}) \Delta \hat{u}_{J}(\boldsymbol{\xi}) d \xi_{m}\right\}+\int_{S^{+}} H_{l K}^{\gamma J}(\boldsymbol{x}, \boldsymbol{\xi}) \Sigma \hat{t}_{J}(\boldsymbol{\xi}) d A(\boldsymbol{\xi})
\end{aligned}
$$

where $D_{m}(\cdot)=n_{i} \varepsilon_{i s m} \partial(\cdot) / \partial \xi_{s}$ is the surface differential operator and $\partial S^{+}$denotes the boundary of $S^{+}$. For a typical case where the surface flux is fully prescribed on the discontinuity surface, both the sum of and the jump in the state variable are unknown a priori and the closure condition $\Delta \hat{u}_{J}=0$ is satisfied on $\partial S^{+}$. The weak-form boundary integral equations for this particular case can be established as follows. By forming the limit $\boldsymbol{x} \rightarrow \boldsymbol{y} \in S^{+}$of (20) and (21) along with the closure condition, then multiplying the results by an arbitrary, smooth test function, and finally integrating certain integrals by parts via Stokes' theorem, it leads to

$$
\begin{aligned}
\frac{1}{2} \int_{S^{+}} \tilde{t}_{P}(\boldsymbol{y}) \sum \hat{u}_{P}^{\gamma}(\boldsymbol{y}) d A(\boldsymbol{y})= & \int_{S^{+}} \tilde{t}_{P}(\boldsymbol{y}) \int_{S^{+}} U_{J}^{\gamma P}(\boldsymbol{\xi}, \boldsymbol{y}) \sum \hat{t}_{J}(\boldsymbol{\xi}) d A(\boldsymbol{\xi}) d A(\boldsymbol{y}) \\
& -\int_{S^{+}} \tilde{t}_{P}(\boldsymbol{y}) \int_{S^{+}} H_{i J}^{\gamma P}(\boldsymbol{\xi}, \boldsymbol{y}) n_{i}^{+}(\boldsymbol{\xi}) \Delta \hat{u}_{J}(\boldsymbol{\xi}) d A(\boldsymbol{\xi}) d A(\boldsymbol{y}) \\
& +\int_{S^{+}} \tilde{t}_{P}(\boldsymbol{y}) \int_{S^{+}} G_{m J}^{\gamma P}(\boldsymbol{\xi}, \boldsymbol{y}) D_{m} \Delta \hat{u}_{J}(\boldsymbol{\xi}) d A(\boldsymbol{\xi}) d A(\boldsymbol{y}) \\
\frac{1}{2} \int_{S^{+}} \tilde{u}_{K}(\boldsymbol{y}) \Delta \hat{t}_{K}^{\gamma}(\boldsymbol{y}) d A(\boldsymbol{y})= & \int_{S^{+}} \tilde{u}_{K}(\boldsymbol{y}) \int_{S^{+}} H_{l K}^{\gamma J}(\boldsymbol{y}, \boldsymbol{\xi}) n_{l}^{+}(\boldsymbol{y}) \sum \hat{t}_{J}(\boldsymbol{\xi}) d A(\boldsymbol{\xi}) d A(\boldsymbol{y}) \\
& -\int_{S^{+}} D_{t} \tilde{u}_{K}(\boldsymbol{y}) \int_{S^{+}} C_{m J}^{\gamma t K}(\boldsymbol{\xi}, \boldsymbol{y}) D_{m} \Delta \hat{u}_{J}(\boldsymbol{\xi}) d A(\boldsymbol{\xi}) d A(\boldsymbol{y}) \\
& -\int_{S^{+}} D_{t} \tilde{u}_{K}(\boldsymbol{y}) \int_{S^{+}} G_{t K}^{\gamma J}(\boldsymbol{y}, \boldsymbol{\xi}) \sum \hat{t}_{J}(\boldsymbol{\xi}) d A(\boldsymbol{\xi}) d A(\boldsymbol{y})
\end{aligned}
$$


where $\tilde{t}_{P}$ and $\tilde{u}_{K}$ are smooth test functions; $\Sigma \hat{u}_{P}^{\gamma}(\boldsymbol{y}) \equiv \hat{u}_{P}^{\gamma}\left(\boldsymbol{y}^{+}\right)+\hat{u}_{P}^{\gamma}\left(\boldsymbol{y}^{-}\right)$; and $\Delta \hat{t}_{K}^{\gamma}(\boldsymbol{y}) \equiv \hat{t}_{K}^{\gamma}\left(\boldsymbol{y}^{+}\right)-\hat{t}_{K}^{\gamma}\left(\boldsymbol{y}^{-}\right)$. It is remarked that the test function $\tilde{u}_{K}$ is chosen to also satisfy the closure condition, i.e. $\tilde{u}_{K}=0$ on $\partial S^{+}$. In addition, both the weak-form boundary integral equations (22) and (23) contain only weakly singular kernels of $\mathcal{O}(1 / r)$ and are sufficient for determining the unknown state-variable data on the surface of discontinuity, i.e. $\Sigma \hat{u}_{P}^{\gamma}$ and $\Delta \hat{u}_{J}$.

\section{Solution procedure}

The weak-form boundary integral equation for the surface flux (23) contains the prescribed information $\Delta \hat{t}_{K}^{\gamma}$ and $\Sigma \hat{t}_{J}$ and is clearly independent of the unknown data $\Sigma \hat{u}_{P}^{\gamma}$. This equation is solved first to obtain the unknown jump $\Delta \hat{u}_{J}$ and this can be accomplished by utilizing standard, weakly singular, symmetric Galerkin boundary element method (e.g. [8, 14]). Various components such as the discretization, approximation of the near-front field, singular and nearly singular double surface integrations, and the numerical evaluation of kernels are properly considered to significantly enhance the accuracy and computational efficiency of the numerical technique. Once the data $\Delta \hat{u}_{J}$ is determined, it supplies (22) as the known information in addition to $\Sigma \hat{t}_{J}$. The sum of the state variable $\Sigma \hat{u}_{P}^{\gamma}$ can, therefore, be obtained by solving the weak-form equation (22) using standard Galerkin method. It is apparent from the above solution procedure and the form of the integral equations that the discretization of (22) and (23) leads to two symmetric systems of linear algebraic equations.

Once $\Sigma \hat{u}_{P}^{\gamma}$ and $\Delta \hat{u}_{J}$ are determined, the state variable and the body flux at any interior point of the body can readily be obtained from the boundary integral relations (20) and (21), respectively. Other essential data such as the intensity factors contained in the singular term and the first non-singular term in the expansion of the body flux in the neighborhood of the boundary of discontinuity surface can also be extracted. In particular, techniques analogous to those proposed by [15] and [16] are employed along with the solved data $\Delta \hat{u}_{J}$ and $\Sigma \hat{u}_{P}^{\gamma}$ to compute the intensity factors and the first non-singular term, respectively.

\section{Numerical results}

As a means for verifying the integral formulation and the implemented solution procedure, a set of boundary value problems under various scenarios are solved and results, when benchmarked with reliable reference solutions, exhibit good agreement. To demonstrate both the accuracy and the convergence of the proposed technique, results of a representative problem associated with linear elasticity (i.e. $\alpha=1$ and $\beta=0$ ) is only presented, here, for brevity.

Consider a penny-shaped crack of radius $a$ and oriented vertically in a halfspace with a depth $h$ (measured from the center of the crack to the free surface) as 
shown in Figure 3(a). The half-space is made of a homogeneous, transversely isotropic, linearly elastic material with independent, relative elastic constants given by $E_{1111}=16.09, E_{1122}=3.35, E_{1133}=5.01, E_{3333}=6.10$, and $E_{1313}=3.83$. The crack is subjected to uniform normal traction $\sigma_{0}$ as shown in Figure 3(b) and the crack front is parameterized by $x_{1}=0, x_{2}=a \sin \beta, x_{3}=h-a \cos \beta$ for $\beta \in[0,2 \pi]$. In the analysis, three meshes are adopted as illustrated in Figure 3(c) and the ratio $h / a=1.25$ is employed. The computed stress intensity factors and Tstresses are first normalized and then compared with the reference solution (generated from an equivalent whole space containing a pair of geometrically symmetrical cracks with respect to the plane $x_{3}=0$ and subjected to the symmetrical loading condition for $\mathrm{BC}_{1}$ and the anti-symmetrical loading condition for $\mathrm{BC}_{2}$ ) as shown in Figures 4 and 5, respectively. It can be seen from this set of results that numerical solutions obtained from the proposed technique are highly accurate and weakly dependent on the level of mesh refinement. In particular, results generated by the coarse and intermediate meshes are nearly indistinguishable from the benchmark solutions. This high quality of numerical solutions results directly from the use of special interpolation functions (e.g. [8, 14]) to capture the near-front relative crack-face displacement.

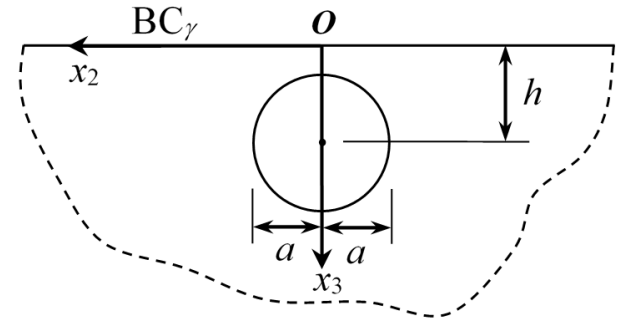

(a)

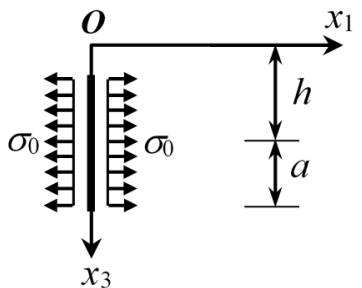

(b)

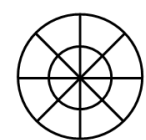

Mesh 1

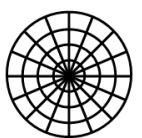

Mesh 2

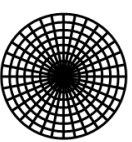

Mesh 3

(c)

Figure 3: (a) Vertical penny-shaped crack in half-space, (b) crack under uniform normal traction, and (c) three meshes adopted in the analysis.

\section{Conclusions}

A set of singularity-reduced boundary integral relations/equations has been established for a half-space containing a surface of discontinuities and subjected to symmetrical and anti-symmetrical boundary conditions on the free surface. The present work has offered not only the formulation derived in a sufficiently broad 


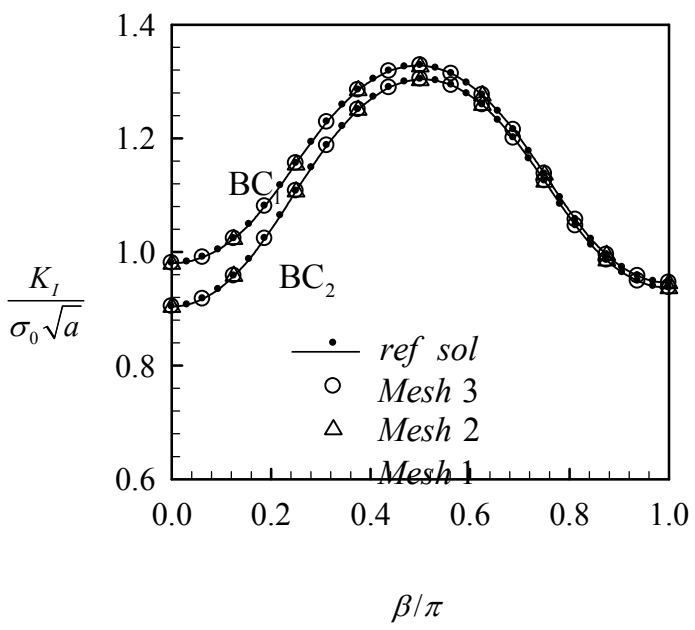

Figure 4: Normalized stress intensity factors for vertical penny-shaped crack.

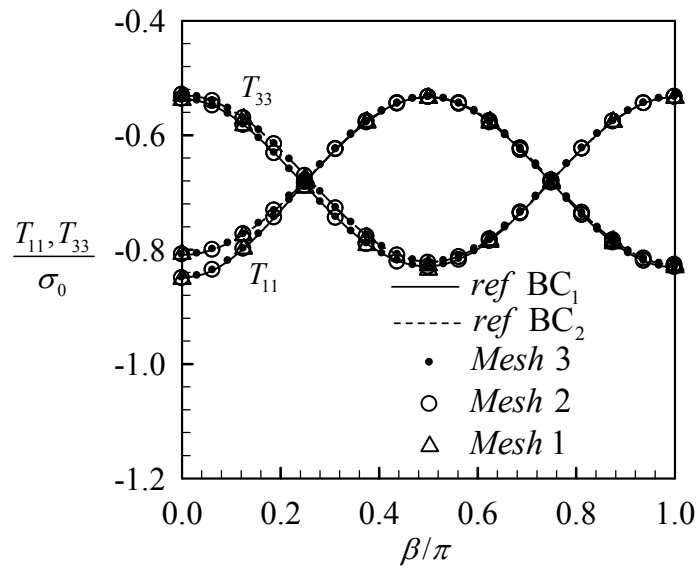

Figure 5: Normalized T-stresses for vertical penny-shaped crack.

framework allowing the treatment of various multi-field boundary value problems but also an efficient and accurate numerical procedure for the analysis of a halfspace containing cracks and discontinuities. An extensive numerical study has confirmed that the proposed numerical scheme is highly accurate, computationally efficient, and robust. It should be remarked that the half-space considered in the present study is a reduced problem of a whole space possessing a plane of symmetry or anti-symmetry. As a result, the number of degrees of freedom can be reduced by a half if the proposed technique is employed. In addition, results established in the present study are fundamental and essential for the development of singularity-reduced boundary integral equations to treat other types of boundary conditions on the surface of the half-space. 


\section{Acknowledgement}

The authors are very grateful for the financial support from the JICA Project for AUN/SEED-Net Scholarship.

\section{References}

[1] Bogdanov, V.L., Nonaxisymmetric problem of the stress-strain state of an elastic half-space with a near-surface circular crack under the action of loads along it. Journal of Mathematical Sciences, 174, pp. 341-366, 2011.

[2] Feng, X.Q., Xu, M., Wang, X. \& Gu, B., Fracture mechanics analysis of three-dimensional ion cut technology. Journal of Mechanics of Materials and Structures, 2(9), pp. 1831-1852, 2007.

[3] Gordeliy, E. \& Detournay, E., Displacement discontinuity method for modeling axisymmetric cracks in an elastic half-space. International Journal of Solids and Structures, 48, pp. 2614-2629, 2011.

[4] Hrylyts'kyi, M.D., Laushnyk, I.P. \& Stankevych, O.M., Reduction of the problem of interaction of cracks in a restrained half space to boundary integral equations. Materials Science, 39(1), pp. 79-85, 2003.

[5] Khai, M.V. \& Sushko, O.P., Interaction of coplanar surface cracks in a halfspace. Strength of Materials, 26(10), pp. 720-724, 1994.

[6] Li, S., Singularity-reduced integral equations for discontinuities in three dimensional elastic media. PhD Dissertation, University of Texas at Austin, USA, 1996.

[7] Li, S. \& Mear, M.E., Singularity-reduced integral equations for displacement discontinuities in three-dimensional linear elastic media. International Journal of Fracture, 93, pp. 87-114, 1998.

[8] Li, S., Mear, M.E. \& Xiao, L., Symmetric weak-form integral equation method for three-dimensional fracture analysis. Computer Methods in Applied Mechanics and Engineering, 151, pp. 435-459, 1998.

[9] Lo, K.K., Three-dimensional crack in the interior of a half-space. Sijthoff \& Noordhoff International Publishers Alphen aan den Rijn, 9, pp. 435-439, 1979.

[10] Movchan, N.V. \& Willis, J.R., Surface-breaking crack in an elastic halfspace. Journal of Engineering Mathematics, 37, pp. 143-154, 2000.

[11] Murakami, Y., Analysis of stress intensity factors of modes I, II and III for inclined surface cracks of arbitrary shape. Engineering Fracture Mechanics, 22(1), pp. 101-114, 1985.

[12] Noguchi, H. \& Smith, R.A., An analysis of a semi-infinite solid with threedimensional cracks. Engineering Fracture Mechanics, 52(1), pp. 1-14, 1995.

[13] Rungamornrat, J. \& Mear, M.E., Weakly-singular, weak-form integral equations for cracks in three-dimensional anisotropic media. International Journal of Solids and Structures, 45, pp. 1283-1301, 2008. 
[14] Rungamornrat, J. \& Mear, M.E., A weakly-singular SGBEM for analysis of cracks in 3D anisotropic media. Computer Methods in Applied Mechanics and Engineering, 197, pp. 4319-4332, 2008.

[15] Rungamornrat, J. \& Senjuntichai, T., Regularized boundary integral representations for dislocations and cracks in smart media. Smart Materials and Structures, 18, 074010, 2009.

[16] Subsathaphol, T., Analysis of T-stress for cracks in 3D linear piezoelectric media. Master Thesis, Chulalongkorn University, Thailand, 2013.

[17] Sushko, O.P. \& Khai, M.V., Solution of problems of the theory of elasticity for a half-space with planar boundary cracks. Journal of Mathematical Sciences, 79(6), pp. 1434-1438, 1996. 